\title{
Ovarian Dysgerminoma: Unusual Presentation in Pregnancy with Acute Abdomen due to Internal Hemorrhage
}

\author{
Agrawal A, ${ }^{1}$ Rijal P, ${ }^{1}$ Pokharel H, ${ }^{1}$ Karki S, ${ }^{2}$ Basnet $\mathbf{P},{ }^{1}$ Dahal $\mathbf{P}^{1}$ \\ ${ }^{1}$ Dept of Obs/Gyn, ${ }^{2}$ Dept of Pathology, BPKIHS
}

\begin{abstract}
Dysgerminoma tends to occur in young women although its incidence in pregnancy is low. Torsion and incarceration occurs in nearly half of the cases. Early diagnosis and appropriate treatment offers the best prognosis to the patient presenting with dysgerminoma. However because of enlarging abdominal size, pregnancy may mask the rapidly growing dysgerminoma often leading to late presentation.

This is a case report of a female 30 years of age with stage $3 \mathrm{C}$ dysgerminoma which lead to acute surgical abdomen at 33 weeks of pregnancy.
\end{abstract}

Keywords : Acute abdomen, dysgerminoma, pregnancy with ovarian mass

\section{INTRODUCTION}

Dysgerminoma, which accounts for $1-5 \%$ of all ovarian cancers, is the most commonly occurring malignant germ cell tumor contributing to $28 \%$ of it. ${ }^{1}$ It tends to occur in young women, though the incidence of pregnancy with dysgerminoma is extremely low. The rate of malignant tumors in the total number of ovarian tumors associated with pregnancy was reported from $1.3 \%$ to $7.9 \% .^{2}$ Torsion and incarceration are common complications among these rapidly enlarging tumors. Obstetrical complications occurred in nearly half and fetal demise occurred in one quarter of the reviewed cases. ${ }^{3}$

The 5 -year survival rate is $96 \%$ if the tumor is confined

\section{CORRESPONDENCE}

Dr Ajay Agrawal

Department of Obstetrics and Gynaecology

Assistant Professor, BPKIHS

Phone: 9852049451

Email: drajayagrawal@yahoo.com to the ovary and $63 \%$ if extension occurs beyond the ovaries. Pregnancy does not alter the prognosis of most ovarian malignancies, but complications such as torsion and rupture may increase the incidence of spontaneous abortion or preterm delivery. ${ }^{4}$

\section{CASE}

A 30 years lady at 31 weeks and 5 days period of gestation referred from a primary health centre of Morang district with ultrasound diagnosis of bilateral adnexal mass with pregnancy was admitted on $25^{\text {th }}$ June 2011 . She was unbooked immunized G2 P1 L1 with previous uneventful pregnancy with normal vaginal delivery. She gave history of dull aching lower abdominal pain and gradually increasing abdominal distension since two weeks. She also had occasional burning micturition with increased frequency and fever. She did not have history of loss of weight and 
appetite. There was no family history of gynecologic malignancy. There was no significant past medical or surgical history. On examination she was thin built and looked pale. She had fever of $100^{\circ} \mathrm{F}$. Her respiratory and cardiovascular systems were unremarkable. Abdomen was grossly distended with fullness in bilateral flanks. Uterus was 34 weeks size with single live fetus in longitudinal lie and cephalic presentation. There was huge mass of variegated consistency of around $15 \times 20 \mathrm{~cm}$ with ill defined margin, restricted mobility, nontender on right side of gravid uterus occupying whole of right hypochondrium and similar but smaller mass of $10 \times 8 \mathrm{~cm}$ in left flank. There was no renal angle tenderness or palpable kidneys.

On investigation her hemoglobin was $7.4 \mathrm{mg} /$ $\mathrm{dl}$, urine culture had growth of E.Coli sensitive to sulbactam+cefoperazone, with which she responded and her fever subsided and other routine investigations were within normal limit. However her abdominal pain did not subside. She was transfused with 3 units of whole blood. She was given steroid coverage for fetal lung maturity. An ultrasonography done on $1^{\text {st }}$ July 2011 revealed large abdominopelvic mass mostly solid in nature, $23 \times 14 \mathrm{~cm}$ in right side of abdomen and another solid mass $13 \times 8.3$ $\mathrm{cm}$ in pouch of Douglas with minimal ascites suggestive of bilateral adnexal mass with a single live fetus of 31-33 weeks of gestation without any malformations. Additional evaluation for serum CA125, AFP and $\beta$ HCG was normal.

On $5^{\text {th }}$ July, at 33 weeks of pregnancy she complained of increasing abdominal pain which was continuous and generalized to whole abdomen not relieved with analgesic. So with a diagnosis of twisted or ruptured ovarian mass she was planned for lower segment caesarean section with removal of ovarian mass and surgical staging. After general anesthesia, midline laparotomy incision was made. There was hemoperitoneum of 2 liters with a superficial tumoral vessel actively bleeding. A lower segment caesarean section was first performed.

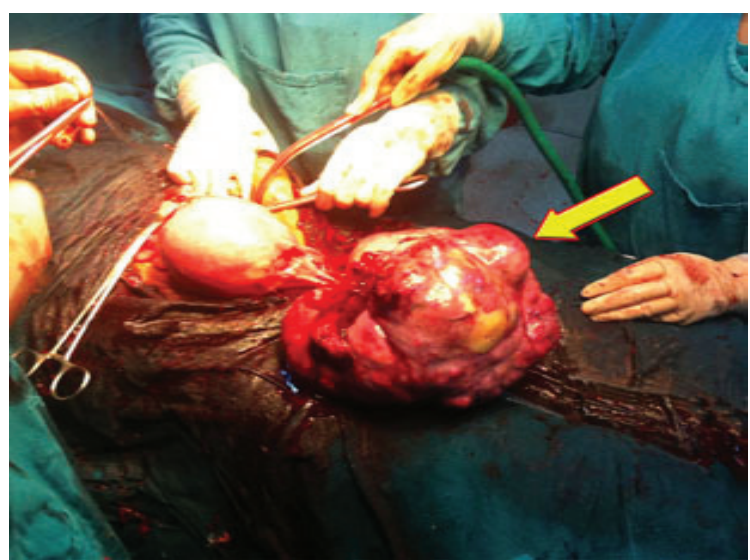

Fig 1(a). Intraoperative finding of huge ovarian mass with the twisted pedicle.

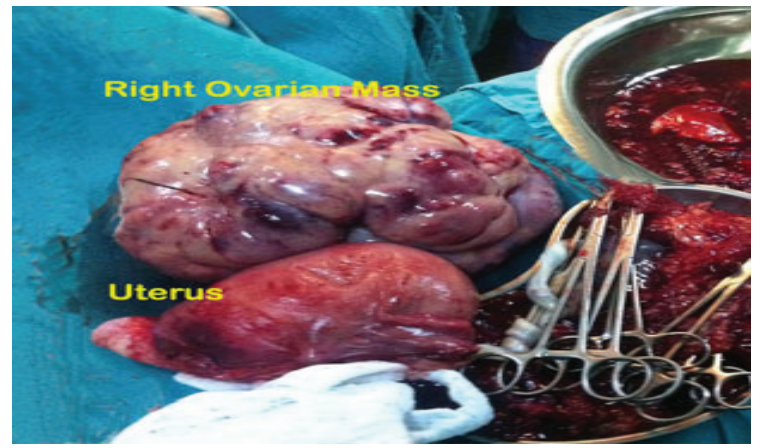

Fig 1 (b). Post partum uterus with ovarian dysgerminoma with omentum

Bosselated huge mass of $25 \times 23 \times 20 \mathrm{~cm}$ was found originating from right ovary and twisted around its pedicle with mostly solid component extending from pouch of Douglas to undersurface of liver. There were multiple metastatic deposits over parietal abdominal peritoneum and omentum with dense adhesion to serosa of sigmoid colon. No deposits were noted on surface of liver. ( Figure 1). Mass was removed after haemostatic division of the attachments and pedicle. The left ovary $(4 \times 1.5 \times 1 \mathrm{~cm})$, fallopian tube and uterus were grossly normal in contrast to sonography diagnosis of bilateral ovarian mass. Since she had no desire for future fertility which was discussed with her before taking her for laparotomy, a total hysterectomy and bilateral salpingo-oophorectomy, infracolic omentectomy with biopsy from parietal wall was then performed. She delivered a preterm baby of 2 $\mathrm{kg}$ with apgar score of 7/10,8/10 at 1 and 5 minutes of life. Abdomen was closed with a drain in the pelvis. Post operative period was uneventful. Sutures were removed on $8^{\text {th }}$ post op day and drain was removed on $10^{\text {th }}$ post operative day.

Histopathology from right ovary revealed features of malignant germ cell tumor having islands of large malignant cells separated by scanty fibrous stroma and infiltrated by lymphocytes, with areas of hemorrhages compatible with ovarian dysgerminoma. Uterus, Left ovary and both fallopian tubes were histologically unremarkable. Sections from omentum and abdominal wall also showed histologic features of tumor deposits (Fig 2a,2b).

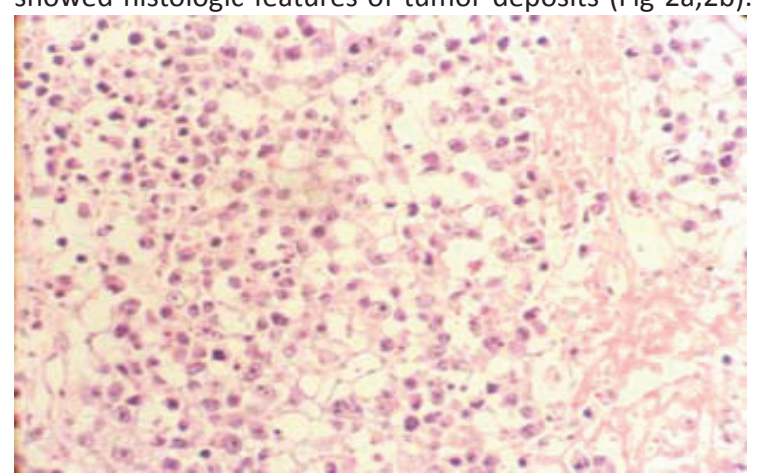

Fig 2(a). Microscopically islands of large malignant cells separated by scanty fibrous stroma 


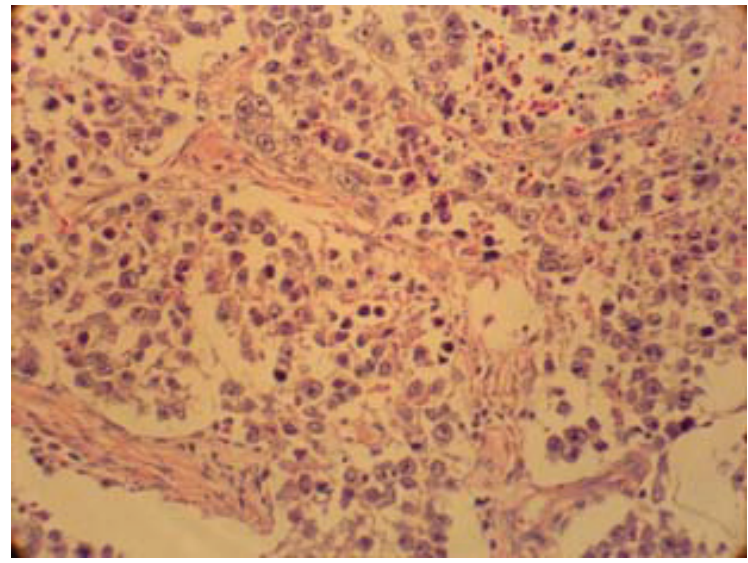

Fig 2(b). Infiltrated by lymphocytes, with areas of haemorrhage

The patient was assigned FIGO Stage 3 C ovarian malignancy and referred to Bharatpur Cancer Hospital for platinumbased chemotherapy.

\section{COMMENT}

Association of ovarian carcinoma with pregnancy is a rare occurrence. It may also present as acute abdomen because of torsion (tumor twisting around its pedicle) or intra-abdominal hemorrhage (from congestion and rupture of superficial tumor vessels) as seen in this patient. ${ }^{5}$ Early diagnosis and appropriate treatment offers the best prognosis to the patient. Bilateral tumors occur in up to $10-15 \%$ of cases. ${ }^{6,7}$ Lee reported mean age of 23.8 years (range $4-63$ years). ${ }^{8}$ Dysgerminoma is also very sensitive to radiation while other germ cell tumors are not. ${ }^{9}$ Chemotherapy and/or radiation can be used to treat patients with metastatic disease. ${ }^{5}$ For patients with early stage disease, cure rates approach $100 \%$, while for those with advanced-stage disease it is least $75 \% .^{9}$ Preoperative diagnosis depends mainly on age, abdominal symptoms, size and consistency of the tumor, and elevated tumor markers. Our patient had late presentation as she was misguided by the increase in abdominal size thinking it to be due to pregnancy. The presentation is usually with chronic abdominal pain and palpable abdominal tumour. Some may present with vaginal bleeding, amenorrhea or precocious puberty. ${ }^{9}$

Elevated tumor markers may assist in determining the diagnosis preoperatively. ${ }^{4}$ Hoei-Hansen et al reported the expression of following markers in ovarian dysgerminomas: placental alkaline phosphatase (PLAP) in 100\% cases, stem cell-related markers OCT-3/4 and KIT in $80 \%$ cases, and NANOG and AP- $2 \gamma$ in $50 \%$ cases. ${ }^{4}$ Kawai et al reported positive rate of AFP in $11.8 \%$ dysgerminoma, and CA 125 in over $50 \%$ cases; they also found a high positive rate of LDH. ${ }^{10}$ In this case CA125, AFP and $\beta$ HCG levels were normal.
Surgery is the first step of management followed by adjuvant chemotherapy (depending on histopathology) 9. Appropriate surgical treatment for patients where fertility needs to be preserved consists of laparotomy with unilateral salpingo-oophorectomy and resection of all visible disease. ${ }^{7,11} \mathrm{~A}$ total abdominal hysterectomy is not mandatory, but may be performed concurrently if patient does not desire future pregnancy as in this case. The current standard adjuvant chemotherapy consists of 3 cycles of BEP. ${ }^{8,11}$ Four courses of BEP are recommended in case of bulky residual tumor after surgery. ${ }^{4,11}$ Recurrence in contralateral ovary can develop over the next 2 years. ${ }^{7}$ Fertility sparing treatments are considered safe in patients with pure ovarian dysgerminomas. ${ }^{12}$

\section{CONCLUSION}

Ovarian cancer very rarely presents as an acute abdomen in pregnancy. However, ovarian malignant dysgerminoma may present as an acute abdomen because of torsion, passive blood congestion, rupture of superficial tumoral vessels and subsequent intra-abdominal hemorrhage. Ovarian dysgerminoma should also be part of the differential diagnosis in a young pregnant woman with acute surgical abdomen and a solid heterogeneous pelvic mass detected by ultrasonographic scan.

\section{REFERENCES}

1. Kdous M, Hachicha R, Gamoudi A, Boussen H, Benna F, Rahal K. Pure dysgerminoma of the ovary. 12 case reports. Tunis Med. 2003;81(12):937-43

2. Creasman WT, Rutledge F, Smith JP. Carcinoma of the ovary associated with pregnancy. Obstet Gynecol. 1971;38(1):111-6.

3. Karlen JR, Akbari A, Cook WA. Dysgerminoma associated with pregnancy. Obstet Gynecol. 1979;53(3):330-5.

4. $\mathrm{Lu} \mathrm{KH}$, Gershenson DM. Update on the management of ovarian germ cell tumours. J Reprod Med. 2005;50(6):417-25

5. Varras M, Tsikini A, Polyzos D, Samara C, Akrivis C. Internal hemorrhage caused by a twisted malignant ovarian dysgerminoma: ultrasonographic findings of a rare case and review of the literature. Clin Exp Obstet Gynecol. 2004;31(1):73-8.

6. Hoei-Hansen CE, Kraggerud SM, Abeler VM, Kaern J, Rajpert De Meyts E, Lothe RA. Ovarian dysgerminomas are characterised by frequent KIT mutations and abundant expression of pluripotency markers. Mol Cancer. 2007;6:12.

7. Behtash N, Zarchi MK. Dysgerminoma in three patients with Swyer syndrome. World J Surg Oncol. 2007;5:71.

8. Lee KH, Lee $\mathbf{I H}$, Kim BG, Nam JH, Kim WK, Kang SB et al. Clinicopathologic characteristics of malignant germ cell tumours in the ovaries of Korean women: a Korean Gynecologic Oncology Group Study. Int J Gynecol Cancer. 2009;19:84-7.

9. Aziz MF. Current management of malignant germ cell tumour of the ovary. Gan To Kagaku Ryoho. 1995;22(Suppl 3):262-76.

10. Kawai M, Kano T, Kikkawa F, Morikawa Y, Oguchi H, Nakashima N et al. Seven tumour markers in benign and malignant germ cell tumours of the ovary. Gynecol Oncol. 1992;45(3):248-53.

11. Pectasides D, Pectasides E, Kassanos D. Germ cell tumours of the ovary. Cancer Treat Rev. 2008;34(5):427-41.

12. Boran N, Tulunay G, Caliskan E, Kose MF, Haberal A. Pregnancy outcomes and menstrual function after fertility sparing surgery for pure ovarian dysgerminomas. Arch Gynecol Obstet. 2005;271(2): 104-8. 